\title{
El cigarrillo electrónico podría contribuir a la cesación tabáquica aunque permanece incierta su seguridad a largo plazo
}

\author{
E-cigarettes may contribute to smoking cessation, although long-term safety remains unclear
}

\section{Comentado de:}

Hartmann-Boyce J, et al. Cochrane Database Syst Rev. 2021;9(9):CD010216. doi: 10.1002/14651858.CD010216.pub6. PMID: $34519354^{1}$

\section{Objetivo}

Evaluar la efectividad, tolerancia y seguridad de los cigarrillos electrónicos (CE), con o sin nicotina (CEN y CESN, respectivamente) para lograr la abstinencia tabáquica a largo plazo.

\section{Métodos}

Revisión sistemática viva y meta-análisis con métodos Cochrane, cuya fuentes de datos fueron el registro especializado del Cochrane Tobacco Addiction Group, el Cochrane Central Register of Controlled Trials (CENTRAL), MEDLINE, Embase y PsycINFO, hasta mayo de 2021

Los criterios de inclusión fueron ensayos clínicos controlados aleatorizados (ECCA), paralelos o cruzados, comparando CE versus control para la abstinencia de cigarrillos en el seguimiento de seis a 12 meses, o en los datos de seguridad entre el mes y los 12 meses de seguimiento. Dada la escasez de ECCA, también se incluyeron estudios no controlados, en los que todos los participantes recibían CE.

\section{Resultados Principales}

Se incluyeron 61 estudios ( $n=16.759,34$ ECCA), en su mayoría con riesgo de sesgo alto o incierto. Sólo 7/61 fueron considerados con bajo riesgo de sesgo y contribuyeron a los resultados principales (ver Tabla 1). Muy pocos estudios informaron datos sobre otros desenlaces o comparaciones.

Tabla 1. Resultados principales de eficacia y seguridad de las distintas comparaciones vs. cigarro electrónico con nicotina. Nota: CEN: cigarrillo electrónico con nicotina; CESN: cigarrillo electrónico sin nicotina; IC: intervalo de confianza.

\begin{tabular}{|c|c|c|c|c|}
\hline Desenlace & Comparación & Riesgo Relativo (IC 95\%) & $\begin{array}{l}\mathrm{N}^{\circ} \text { de participantes } \\
\text { (Estudios) }\end{array}$ & $\begin{array}{l}\text { Certeza de la } \\
\text { evidencia }\end{array}$ \\
\hline \multirow{3}{*}{$\begin{array}{l}\text { Cesación tabáquica } \\
\text { entre } 6 \text { a } 12 \text { meses }\end{array}$} & CEN vs. terapia de reemplazo nicotínica & $1,53(1,21$ a 1,93$)$ & $1.924(4)$ & Moderada \\
\hline & CEN vs. CESN & $1,94(1,21$ a 3,13$)$ & $1.447(5)$ & Moderada \\
\hline & CEN vs. apoyo conductual/no soporte & $2,61(1,44$ a 4,74$)$ & $2.886(6)$ & Muy baja \\
\hline \multirow{3}{*}{$\begin{array}{l}\text { Efectos adversos } \\
\text { entre } 12 \text { semanas a } \\
6 \text { meses }\end{array}$} & CEN vs. terapia de reemplazo nicotínica & $0,98(0,80$ a 1,19$)$ & $485(2)$ & Baja \\
\hline & CEN vs. CESN & $1,01(0,91$ a 1,11$)$ & $601(3)$ & Moderada \\
\hline & CEN vs. apoyo conductual/no soporte & $1,22(1,12$ a 1,32$)$ & $765(4)$ & Baja \\
\hline \multirow{3}{*}{$\begin{array}{l}\text { Efectos adversos } \\
\text { serios entre } 4 \\
\text { semanas a } 6 \text { meses }\end{array}$} & CEN vs. terapia de reemplazo nicotínica & $1,44(0,94$ a 2,19$)$ & $1.183(3)$ & Baja \\
\hline & CEN vs. CESN & $0,95(0,52$ a 1,72$)$ & $1.033(6)$ & Baja \\
\hline & CEN vs. apoyo conductual/no soporte & $1,51(0,70$ a 3,24$)$ & $1.303(7)$ & Muy baja \\
\hline
\end{tabular}

\section{Conclusiones}

Evidencia de moderada certeza indica que los CEN podrían aumentar las tasas de abandono de cigarrillo convencional en comparación con la terapia de reemplazo nicotínica o los CESN, pero la evidencia que los compara con apoyo conductual exclusivo o sin apoyo es incierta.

Fuente de financiamiento / Conflicto de interés de los autores: Las fuentes principales de financiamiento de esta actualización y las previas fueron un subsidio del Tobacco Advisory Group Cancer Research UK Project Grant, y fondos recibidos del National Institute for Health Research del Reino Unido para el Cochrane Tobacco Addiction Group. Un autor recibió fondos para investigación de CE. Otros autores recibieron financiamientos variados de industria farmacéutica para hablar sobre los fármacos para la cesación tabáquica.

\section{Comentario}

El rol del CE en la cesación tabáquica es un tema polémico y esta revisión sistemática actualizada aumenta la discusión. En Argentina su comercialización está prohibida por la ANMAT, y la Guía de Práctica Clínica Nacional Tratamiento de la Adicción al Tabaco ${ }^{2}$ expide una recomendación en contra de su uso basándose en la versión anterior de esta revisión y en el reporte de eventos adversos serios.

Los autores del meta-análisis resumido calificaron con moderada certeza de evidencia la eficacia del CEN comparado con la TRN o los CESN mediante el sistema GRADE ${ }^{3}$, el cual permite explicitar las fortalezas y falencias de la evidencia. Para el desenlace principal, la abstinencia de cigarrillos, en ambas comparaciones el nivel de certeza fue reducido un nivel por 
imprecisión, al incumplir el criterio de tamaño óptimo de la información (menos de 300 eventos en total). Uno de los cuatro estudios de la comparación CEN con CESN fue considerado como de alto riesgo de sesgo, pero dado que la eliminación de este estudio en el análisis de sensibilidad aumentó la dirección del efecto a favor del CEN, los autores no degradaron la certeza de la evidencia en este dominio. Aunque muchos estudios no fueron ciegos para los participantes y los profesionales y podría haberse disminuido el nivel de evidencia por riesgo de sesgo de desempeño, los autores consideraron un nivel similar de soporte en ambas ramas, por lo que juzgaron el riesgo de este sesgo como improbable.

Un aspecto central en debate es la definición del desenlace de interés, es decir, si el dejar de fumar cigarrillos para pasar a consumir de manera crónica nicotina es el objetivo buscado como marcador de efectividad en las terapias de cesación tabáquica. Los criterios de dependencia de cualquier sustancia implican su uso sostenido y priorizado, y sin dudas, la combustión de cigarrillos asegura la inhalación de cientos de sustancias toxicas. Pero la cesación implica también superar la dependencia de la nicotina, lo que no ocurriría en el caso de persistir el uso de CEN, ya que la nicotina absorbida por vía inhalada genera un pico plasmático en pocos segundos que cumple un rol central en la dependencia a esta sustancia. En el principal estudio de este meta-análisis, el $80 \%$ de los participantes que dejaron el cigarrillo convencional continuó utilizando el CEN al año de seguimiento, en comparación con el $9 \%$ de los participantes en la rama TRN ${ }^{4}$. Otra desventaja de los CE es que una mayor disponibilidad de este tipo de dispositivos podría facilitar significativamente la iniciación tabáquica en los jóvenes y los adolescentes, como lo indican los resultados de un meta-análisis reciente ${ }^{5,6}$.

Por otro lado, con respecto a la seguridad de la intervención evaluada en la revisión de Hartmann-Boyce et al, encontramos una importante limitación que resulta de haber incluido estudios de corto seguimiento para evaluar los efectos del uso crónico. Un evento adverso importante, descrito principalmente entre 2012 y 2019, fue la enfermedad pulmonar grave relacionada al CE (conocida como EVALI por sus iniciales en inglés) que causó miles de muertes en jóvenes ${ }^{7}$. Existen, además, daños colaterales vinculados al uso y tenencia de CE que los estudios de investigación clínica no llegan a reportar, como los incendios, las explosiones del dispositivo, la intoxicación de niños por ingesta de cartuchos con nicotina o su utilización para intentos de suicidio, como se anuncia en la página del gobierno del Reino Unido, uno de los pocos países que avala estos dispositivos para la cesación tabáquica ${ }^{8}$.

\section{Conclusiones de los comentadores}

A pesar que se logre la cesación tabáquica durante el primer año, mantener un consumo crónico de nicotina inhalada del CEN no es dejar de fumar; puede conllevar considerables riesgos de seguridad y, quizás, un retorno al consumo de cigarrillos convencionales por no resultar tan gratificante como el fumar. La falta de evidencia de seguridad a largo plazo hace cuestionable su uso hasta que estos desenlaces sean debidamente determinados.

Brunilda Casetta [ Argentina, Ministerio de Salud de la Nación. brunilda.ministerio@gmail.com ]

Agustín Ciapponi [ Servicio de Medicina Familiar y Comunitaria, Hospital Italiano de Buenos Aires; Centro Cochrane Argentina, Instituto de Efectividad Clínica y Sanitaria. aciapponi@iecs.org.ar ]

Casetta B, Ciapponi A. El cigarrillo electrónico podría contribuir a la cesación tabáquica aunque permanece incierta su seguridad a largo plazo. Evid Actual Pract Ambul. 2021;24(4):e002164. Available from: https://dx.doi.org/10.51987/EVIDENCIA.V25I1.6985. Comentado de: Hartmann-Boyce J, et al. Electronic cigarettes for smoking cessation. Cochrane Database Syst Rev. 2021;9(9):CD010216. doi: 10.1002/14651858.CD010216.pub6. PMID: 34519354

\section{Referencias}

1. Hartmann-Boyce J, Mcrobbie H, Butler AR, et al. Electronic cigarettes for smoking cessation. Cochrane Database Syst Rev. $2021 ; 9(9): C D 010216$. Available from: 10.1002/14651858.CD010216.pub6.

2. Casetta B, Videla AJ. Argentina, Ministerio de Salud de la Nación. Guía de Práctica Clínica Nacional de Tratamiento de la Adicción al Tabaco 2020; 2020. Available from: https://bancos.salud.gob.ar/sites/default/files/2020-05/implementacion-gpcn-tratamiento-adiccion-tabaco.pdf.

3. Guyatt G, Oxman AD, Akl EA, et al. GRADE guidelines: 1. Introduction-GRADE evidence profiles and summary of findings tables. J Clin Epidemiol. 2011;64(4):383-94. Available from: 10.1016/j.jclinepi.2010.04.026.

4. Hajek P, Phillips-Waller A, Przulj D, et al. A Randomized Trial of E-Cigarettes versus Nicotine-Replacement Therapy. N Engl J Med. 2019;380(7):629637. Available from: 10.1056/NEJMoa1808779.

5. Salgado MV. El uso de cigarrillo electrónico se asocia al uso posterior de tabaco entre adolescentes. Comentado de: Yoong SL, et al. Association between electronic nicotine delivery systems and electronic non-nicotine delivery systems with initiation of tobacco use in individuals aged $<20$ years. A systematic review and meta-analysis. PLoS One. 2021;16(9):e0256044. doi: 10.1371/journal.pone.0256044. PMID: 34495974. Evid Actual Pract Ambul. 2021;24(4):e002161. Available from: 10.51987/EVIDENCIA.V25I1.6980;http://www.evidencia.org/index.php/Evidencia/article/view/6980.

6. Yoong SL, Hall A, Turon $\mathrm{H}$, et al. Association between electronic nicotine delivery systems and electronic non-nicotine delivery systems with initiation of tobacco use in individuals aged < 20 years. A systematic review and meta-analysis. PLoS One. 2021;16(9):256044. Available from: 10.1371/journal.pone.0256044.

7. Jonas AM, Raj R. Vaping-Related Acute Parenchymal Lung Injury: A Systematic Review. Chest. 2020;158(4):1555-1565. Available from: 10.1016/ j.chest.2020.03.085.

8. Evidence review of e-cigarettes and heated tobacco products 2018: executive summary; 2018. Available from: https://www.gov.uk/government/ publications/e-cigarettes-and-heated-tobacco-products-evidence-review/evidence-review-of-e-cigarettes-and-heated-tobacco-products-2018executive-summary [Last access: 2021-11-02]. 